\title{
Sessile Serrated Adenomas: Why Conventional Endoscopy Is Okay for Unconventional Polyps
}

\author{
Stephen J. Lanspa $\cdot$ Henry T. Lynch
}

Published online: 16 September 2014

(c) Springer Science+Business Media New York 2014

Sessile serrated adenomas (SSA) are premalignant flat lesions localized predominantly in the proximal colon, which, until recently, had often been unrecognized by the endoscopist [1]. Pereyra et al. [2] report in this issue of Digestive Diseases and Sciences that these lesions can be successfully identified using conventional colonoscopy.

Compared with the understanding of genetic mutations accompanying the more common adenomatous polyps (chromosomal instability pathway), the pattern of DNA alterations present in SSA-related colorectal cancer (CRC) development follows the serrated pathway, likely related to molecular abnormalities that involve CpG islands methylation, referred to as the $\mathrm{CpG}$ island methylator phenotype or CIMP [3]. Activating mutations of the mitogen-activated protein kinase pathway comprise components of the oncogenes $B R A F$ or $K R A S[4,5]$. Since tumor progression to CRC may be accelerated compared with the chromosomal instability pathway, SSAs are hence strongly associated with interval CRC. It is fascinating that these changes at the molecular level could have a phenotypic expression recognizable to the observant endoscopist.

Pereyra et al. [1] reported that these lesions are identifiable by luminal surface features such as excess proximal localization, flat morphology, red surface color, and the presence of a mucus cap, as shown in their paper. Endoscopic presentation is subtle: Polyps in Pereyra' s study were few (1.6 per patient) and small (43\% were $5 \mathrm{~mm}$ or smaller), requiring heightened awareness for identification, which, reassuringly, were recognized without specialized

\section{S. J. Lanspa · H. T. Lynch $(\bowtie)$}

Department of Preventive Medicine and Public Health,

Creighton University, 2500 California Plaza, Omaha, NE 68178,

USA

e-mail: htlynch@creighton.edu endoscopic equipment, facilitating their complete removal and pathological review.

Since SSA progression to CRC can be prevented through polypectomy [1], awareness of these lesions among endoscopists and pathologists is important. Since criteria for pathological diagnosis of SSA are evolving, SSA may be underdiagnosed [6]. As noted in Pereyra' s study, there was initially low inter-observer agreement among the pathologists in the diagnosis of SSA. The endoscopist's ability to alert pathologists about a possible SSA will likely improve surveillance planning.

East et al. [4] discussed the evolution of CRC, calling attention to the concern that most CRCs have their origin in two types of precancerous epithelial polyps: hyperplastic and adenomas. For more than a half century, all colorectal epithelial polyps were assumed to be either adenomatous with malignant potential or hyperplastic, a fundamentally benign lesion without malignant potential. Adenomas progress to carcinomas within a single linear sequence of genetic alterations of the tumor suppressor genes $A P C$, $p 53$, or mismatch repair (MMR) genes as well as oncogenes such as KRAS. Exceptions to these polyp types were reported over time, but received little attention until 1990 when Longacre and Fenoglio-Preiser addressed the concept of an intermediate type of polyp. In their study of more than 18,000 colorectal polyps, $110(0.6 \%)$ were diagnosed as serrated adenomas [7]. Most of these polyps had not been discerned as having aberrant histology. Nevertheless, about one-third had been classified as hyperplastic polyps and another third as atypical adenomas, while final third $(0.2 \%$ of all polyps) were described as "intermediate lesions." Such variant classification obfuscated the correct identification of these lesions: $11 \%$ of the serrated adenomas were severely dysplastic or had foci of intramucosal carcinoma, indicating that these lesions are precancerous 
and may be particularly predisposed to malignant transformation. These serrated adenomas also occurred more frequently in the proximal colon than did either conventional adenomas or hyperplastic polyps. Careful pathologic analysis indicates that serrated adenomas are fundamentally adenomas that resembled hyperplastic polyps due to the serrated or saw-toothed contour of the crypt epithelium, similar to that of hyperplastic polyps [4]. The study demonstrated the existence of mixed hyperplastic and adenomatous polyps, which were interpreted as "chance collisions" between the two recognized types of polyp.

East et al. [4] noted that this concept of a separate serrated pathway indicated the presence of a CRC subtype that did not develop within a pre-existing adenoma. They also demonstrated that hyperplastic polyps and related lesions could fill the gap left by adenoma removal.

What can the present study tell us about other at-risk patients? For one, 70-80\% of CRCs are located in the proximal colon in the Lynch syndrome [8], which was excluded from analysis in this study. Importantly, the Lynch syndrome is by far the most frequent hereditary form of CRC, also featuring multiple extra-colonic cancer sites. The Lynch syndrome, along with proximal CRCs, also includes SSAs, increasing the challenge of endoscopic recognition [9]. Why is there a proclivity for SSAs and CRCs to localize to the proximal colon? Although a unique mucosal susceptibility and alterations of the proximal microbiome have been suggested, causal identification awaits further scientific investigation.

Also excluded from the study were patients carrying a diagnosis of inflammatory bowel disease, familial adenomatous polyposis, or the serrated polyposis syndrome [1]. As endoscopists improve their ability to identify SSA, it will be of potentially great value to understand the involvement of SSA in those conditions, in particular with regard to the pathology, clinical features, and carcinogenesis pathways in comparison with sporadic SSA.

Serrated polyps are believed to be precursors of approximately $15 \%$ of sporadic CRCs [10]. Win et al. [11] reported that family members of individuals who met the clinical criteria for SSA to be at an increased risk of CRC and possibly for pancreatic cancer. Their findings support the contention that a familial serrated polyposis syndrome exists, which can likely be identified using defined criteria. A familial syndrome thus identified using predominantly retrospective clinical data is a prime subject for genetic analysis, which we hope will be accomplished in the near future.

\section{References}

1. Vemulapalli KC, Rex DK. Failure to recognize serrated polyposis syndrome in a cohort with large sessile colorectal polyps. Gastrointest Endosc.. 2012;75:1206-1210.

2. Pereyra L, Gómez EJ, González R, et al. Finding sessile serrated adenomas: Is it possible to identify them during conventional colonoscopy? Dig Dis Sci. (Epub ahead of print). doi: 10.1007/ s10620-014-3295-z.

3. Sweetser S, Smyrk TC, Sinicrope FA. Serrated colon polyps as precursors to colorectal cancer. Clin Gastroenterol Hepatol.. 2013;11:760-767.

4. Jass JR, Biden KG, Cummings M, et al. Characterisation of a subtype of colorectal cancer combining features of the suppressor and mild mutator pathways. J Clin Pathol.. 1999;52:455-460.

5. East JE, Saunders BP, Jass JR. Sporadic and syndromic hyperplastic polyps and serrated adenomas of the colon: classification, molecular genetics, natural history, and clinical management. Gastroenterol Clin North Am.. 2008;37:25-46.

6. Tinmouth J, Henry P, Hsieh E, et al. Sessile serrated polyps at screening colonoscopy: Have they been under diagnosed? Am J Gastroenterol (in press).

7. Longacre TA, Fenoglio-Preiser CM. Mixed hyperplastic adenomatous polyps/serrated adenomas: a distinct form of colorectal neoplasia. Am J Surg Pathol.. 1990;14:524-537.

8. Lynch PM, Lynch HT, Harris RE. Hereditary proximal colonic cancer. Dis Colon Rectum.. 1977;20:661-668.

9. Rondagh EJ, Gulikers S, Gomez-Garcia EB, et al. Nonpolypoid colorectal neoplasms: a challenge in endoscopic surveillance of patients with Lynch syndrome. Endoscopy. 2013;45:257-264.

10. Lanspa SJ, Ahnen DJ, Lynch HT. Serrated polyposis: the last (or only the latest?) frontier of familial polyposis? Am J Gastroenterol. 2012;107:779-781.

11. Win AK, Walters RJ, Buchanan DD, et al. Cancer risks for relatives of patients with serrated polyposis. Am J Gastroenterol. 2012;107:770-778. 